PAPER • OPEN ACCESS

Heated thin film gauge arrangements to reduce uncertainty in transient heat transfer measurements

To cite this article: Inés Usandizaga et al 2021 Meas. Sci. Technol. 32015906

View the article online for updates and enhancements. 


\title{
Heated thin film gauge arrangements to reduce uncertainty in transient heat transfer measurements
}

\author{
Inés Usandizaga, Paul Beard, Kam Chana and Thomas Povey \\ Osney Thermo-Fluids Laboratory, Department of Engineering Science, University of Oxford, Parks Road, \\ Oxford OX1 3PJ, UK \\ E-mail: thomas.povey@eng.ox.ac.uk
}

Received 18 May 2020, revised 15 September 2020

Accepted for publication 12 October 2020

Published 2 November 2020

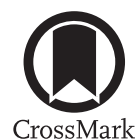

\begin{abstract}
This paper describes the development of heated double-sided thin film gauge configurations for transient heat transfer measurements. By heating the substrate it is possible to measure the heat flux over a range of surface temperatures and deduce the adiabatic wall temperature and the external heat transfer coefficient. The accuracy of the measurement depends on the stability of the regression of heat flux against wall temperature and can be improved by extending the range of wall temperature over which the regression is performed. In this paper we compare two methods of local heating: double-sided gauges with an underside thin film heater and self-heating double-sided gauges. Both arrangements have been used in the Oxford Turbine Research Facility to measure the heat transfer on the uncooled turbine shroud of the MT1 high-pressure turbine stage at engine-representative conditions. These measurements yield improved regressions compared to conventional techniques to determine the adiabatic wall temperature and the heat transfer coefficient.
\end{abstract}

Keywords: heat transfer, thin film gauge, turbine, heat flux, instrumentation

(Some figures may appear in colour only in the online journal)

\section{Nomenclature}

Romans
$A_{\mathrm{g}}$
$c$
$d$
$\mathrm{Gr}$
$h$
$I$
$k$

Gauge area $\left(\mathrm{m}^{2}\right)$

Specific heat capacity $\left(\mathrm{J} \mathrm{kg}^{-1} \mathrm{~K}^{-1}\right)$

Gauge track width (m)

Grashof number $(-)$

Surface heat transfer coefficient $\left(\mathrm{W} \mathrm{m}^{-2} \mathrm{~K}^{-1}\right)$

Current (A)

Substrate thermal conductivity $\left(\mathrm{W} \mathrm{m}^{-1} \mathrm{~K}^{-1}\right.$ ) terms of the Creative Commons Attribution 4.0 licence. Any further distribution of this work must maintain attribution to the author(s) and the title of the work, journal citation and DOI.
Gauge track length (m)

Mach number (-)

Nusselt number (-)

Prandtl number (-)

Heat flux $\left(\mathrm{W} \mathrm{m}^{-2}\right)$

Radius of heating source (m)

Resistance $(\Omega)$

Heating source radius (m)

Stability criterion (-)

Temperature (K)

Temperature at which the temperature coefficient of resistance is determined $(\mathrm{K})$

Gas temperature $(\mathrm{K})$

Time (s)

Voltage (V)

Depth/substrate thickness (m) 


$\begin{array}{ll}\text { Greeks } & \\ \alpha & \text { Temperature coefficient of resistance }\left(\mathrm{K}^{-1}\right) \\ \mathrm{K} & \text { Thermal diffusivity }\left(\mathrm{m}^{2} \mathrm{~s}^{-1}\right) \\ \rho & \text { Density }\left(\mathrm{kg} \mathrm{m}^{-3}\right) \\ \text { Subscripts } & \\ \text { aw } & \text { Adiabatic wall } \\ \text { conv } & \text { Convective } \\ 0 & \text { Evaluated at reference temperature } \\ \mathrm{g} 1 & \text { Upper gauge } \\ \mathrm{g} 2 & \text { Lower gauge } \\ \mathrm{H} & \text { Heater } \\ \mathrm{i} & \text { Initial condition } \\ \text { wi } & \text { Initial wall condition } \\ \text { Acronyms } & \\ \text { HP } & \text { High pressure } \\ \text { HTA } & \text { Heat transfer amplifier } \\ \text { NGV } & \text { Nozzle guide vane } \\ \text { OTRF } & \text { Oxford turbine research facility } \\ \text { TFG } & \text { Thin film gauge } \\ \end{array}$

\section{Introduction}

A number of methods have been developed to determine the heat transfer between surfaces and a fluid, including: thermopiles; Gardon gauges; thermocouples with a thermal resistance layer in-between; calorimeter methods; thermochromic liquid crystals; continuous energy supply methods (e.g. heater foils); mass transfer methods; and platinum resistive gauges. An excellent overview is given by Childs et al [1].

Of the many ways of categorizing these methods, one could consider each technique as primarily suited to steady-state measurements (continuously running facilities) or unsteady measurements (short-duration facilities). The platinum thin film resistive gauge is particularly suited to unsteady measurements in which the surface heat flux is deduced from the temporal surface temperature response. The classical situation is that of a thermally semi-infinite surface surject to a step change in external flow temperature-a case that is possible to solve analytically - but the technique is not limited to this case. The very low thermal resistance of the platinum thin film makes it uniquely suitable in situations where high frequency response is required (up to $100 \mathrm{kHz}$ ).

Although the principles were known much earlier, it was not until the early 1970s that the techniques became more widespread. Platinum gauges spluttered onto ceramic surfaces were used for hypersonic research in the Osney Laboratory in Oxford University [2]. This was followed by the development of techniques to deposit gauges onto flexible polyamide surfaces [3, 4], and on to enamelled metal surfaces [5]. With this came the application of thin film gauges in transient turbine test facilities on both sides of the Atlantic. A history of the development of the thin film gauge is given by Jones [6].

Of particular interest is the development of two type of double-sided gauge: those using two thin film resistance gauges spluttered onto opposing sides of a polyamide film, and primarily used in quasi-steady-state mode [3]; and the socalled direct-heat-flux-gauge composed of a thin film on polyamide with underside thermocouple, analysed as a compound thermal system [7].
One advantage of the double-sided gauge is that it does not require a component-scale 1D assumption of the heat conduction into the wall, and it is possible to process data without knowing the thermal properties of the underlying surface. On a thin-walled component, such as a cooled aerofoil the assumption of a semi-infinite substrate is usually not valid, making the processing of data from single-sided gauges highly complex due to the need to account for lateral conduction effects and underside heating.

More recently_following work by Anthony et al [8] and Thorpe et al [9] — the Osney Laboratory has been active in miniaturizing thin film gauge technology allowing greater spatial resolutions to be achieved [10].

\section{Calibration}

Use of thin-film gauges to measure heat transfer based on surface temperature response requires calibration of both the effective temperature coefficient of resistance of the gauge and of the substrate thermal properties. Depending on the construction (single semi-infinite substrate; gauge over semi-infinite substrate, etc) and mode of operation of the gauge, the thermal properties of one or more layers may need to be characterised. This is typically done using pulsed laser heating [11], pulsed resistive self-heating [12], radiant heating [12], or with a heated free jet [7] and reference gauge (calorimeter, for example).

In the present study we take the calibration for a proprietary polyamide material with glue backing (Kapton with $3 \mathrm{M}$ glue layer) from the study of Piccini et al [7]. Gauges were individually calibrated, as discussed in a later section.

\section{Regression methods}

In recent years there has been an increasing interest in the study of cooled engine parts, including the measurement of film effectiveness and cooling flow redistribution. In many scaled experiments, regions of high film effectiveness are particularly challenging for heat transfer measurements due to the low driving temperature difference between gas and wall, which makes regressions of heat flux as a function of wall temperature (performed to determine the adiabatic wall temperature and the heat transfer coefficient) less stable. In these regions, methods for artificially varying the wall temperature (by heating or cooling) can be used to improve the stability of the regression, i.e. increase the temperature range, enhancing the accuracy with which the adiabatic wall temperature and heat transfer coefficient and can be measured.

Improving the accuracy of this regression is essential for experiments in which the film effectiveness is to be determined. There are three key challenges for the measurement of the heat transfer on cooled engine parts using thin film gauges: to increase the spatial resolution of the measurements by improving the manufacturing technology, to reduce the uncertainties arising from the assumption of a simplified geometry to model the thermal conduction in the gauge 
substrate (e.g. a semi-infinite layer), and to develop methods to accurately determine the Nusselt number and film cooling effectiveness in regions with low heat transfer driving temperature difference.

Two particular interesting studies which could be regarded as improved regression methods, are those of Xue et al [13] and Collins et al [14]. In the work of Xue et al [13], the authors propose a dual linear regression technique to allow simultaneous determination of: recovery temperature; heat transfer coefficient; and film cooling effectiveness. Two experiments are required, with sufficient difference in the coolant-tomainstream temperature ratio between experiments (all governing non-dimensional parameters are assumed to remain unchanged). In the work of Collins et al [14], the authors introduce three regression concepts to improve processing in turbine experiments: floating point regression as a means of correcting for heat transfer or work (according to simple theoretical model) between a driving gas temperature measurement point and the location of the surface of interest; multiple wall temperature processing using a pre-heated wall at various temperatures (see also $[15,16]$ ) to extend the regression range; and a phase-locked local regression technique in which both high frequency $(\sim 150 \mathrm{kHz})$ and low frequency $(\sim 1 \mathrm{~Hz})$ transients are used to determine the local heat transfer coefficient and adiabatic wall temperature.

\section{The present study}

In this paper two new gauge concepts are presented, which combine double-sided thin film gauges with the possibility to heat the substrate layer and thus improve the stability of the regression. The advantage of locally heating the gauge substrate instead of changing the temperature of the entire turbine part is that the heating system is not limited by spatial constrains in the part. Moreover, it is difficult to achieve the uniform initial surface temperature required to carry out heat transfer measurements, given the thermal losses to the ambient and neighbouring components due to insulation difficulties. With heated gauges an improved regression can also be obtained without the need for complex heating systems. The concepts sit in the same landscape as other multiple wall temperature processing techniques [14-16], but are-in principle at least-simpler in implementation.

\section{Development of double-sided TFGs for heat transfer measurement}

The theory of thin film surface resistance gauges for heat transfer measurements is well documented [17]. The resistance of a thin film gauge increases linearly with temperature:

$$
R(T)=R_{0}(1+\alpha \Delta T)
$$

where $R_{0}$ is the resistance at a reference temperature $T_{0}$ and $\alpha$ is the temperature coefficient of resistance. Defining the change in the resistance of the thin film gauge $\Delta R=R(T)-R_{0}$ and the change in surface temperature $\Delta T=T-T_{0}$ it follows that

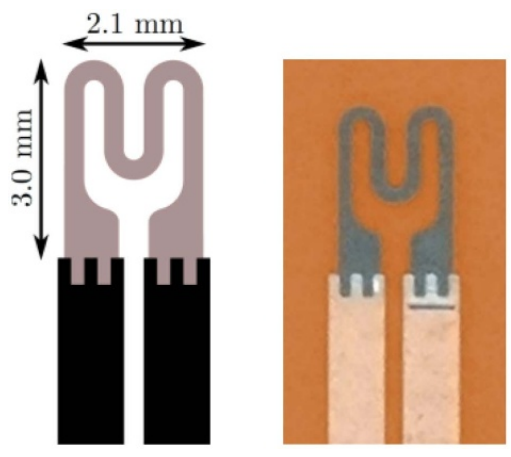

Figure 1. Gauge dimensions and photograph of gauge.

$$
\Delta T=\frac{\Delta R}{\alpha R_{0}}
$$

For a constant gauge current, $I$, the temperature history of the gauge can be obtained by recording the potential difference $\Delta \mathrm{V}$ across the thin film gauge.

In a short-duration test the penetration depth of the thermal pulse into the insulating layer is small compared to other dimensions and hence the insulating layer can be taken to be homogenous and isotropic in the lateral directions. In particular, the heat flux into the wall is much larger than any lateral heat flux. Assuming furthermore that the effect of the gauge is negligible, i.e. that it has negligible thermal resistance, the equation governing the temperature distribution in the insulating layer is the 1D Fourier equation

$$
\frac{\partial^{2} T}{\partial x^{2}}=\frac{1}{\kappa} \frac{\partial T}{\partial t}
$$

with the thermal diffusivity $\kappa=k / \rho c$.

As long as the penetration depth of the thermal pulse is sufficiently small that a semi-infinite analysis can be performed, this equation can be used to infer the surface heat flux from the surface temperature [17]. While it is difficult to calculate the time-dependent heat flux analytically, the surface temperature signals can be processed into heat transfer rates by the use of analogue electronic circuits [18]. More recently, impulse response pro-cessing of temperature signals has been introduced [19] (see appendix A).

The design of the thin film gauges employed in this study is based on the work by Collins et al [10]. The aim of the gauge design is to optimise the gauge sensitivity by maximising the $l / d$ ratio of the thin film, where $l$ is the gauge length and $d$ the width of the gauge. The ratio can be increased through the use of long thin films in serpentine patterns in order to increase spatial resolution. The final gauge design and the manufactured result are shown in figure 1.

Details on the manufacturing process are given by Collins et al [10]. Double-sided thin film gauges can be fabricated from a pair of single-sided thin film gauges glued together. The Kapton and glue layers were found to have very similar thermal properties and will be regarded as one layer in the following. The optimum thickness of the insulating layer between the top and bottom gauges was determined as approximately 


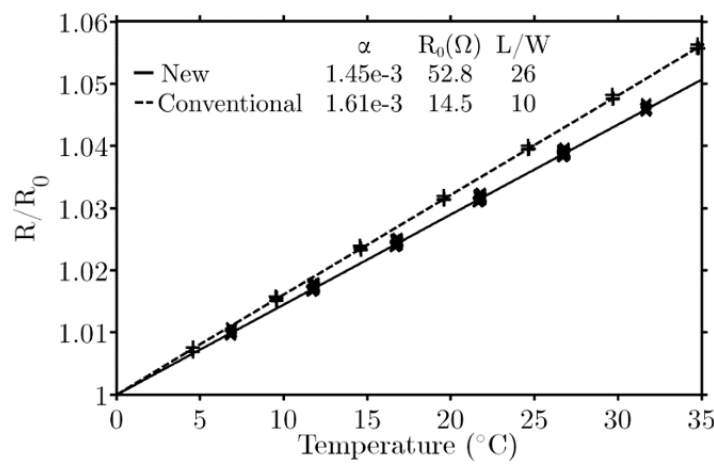

Figure 2. Comparison of calibration of conventional and new Oxford TFHFGs (from [10]).

$100 \mu \mathrm{m}$ based on the modelling of the thermal pulse through the double-sided gauge [20].

The thin film gauges were calibrated in a water bath. In the calibration, the resistance of the gauges was measured at different temperatures to obtain the temperature coefficient of resistance $\alpha$. The effect of changes in lead resistance on the temperature coefficient prior to the test was accounted for.

The gauges used in this study were from the same manufacturing process - and batch - as those used in [10], for which typical calibration curves (from [10]) are given in figure 2. For our purpose it is sufficient to note good linearity, and good repeatability over a wide range of temperature for a number of temperature cycles. A detailed discussion of calibration issues and underlying physics is presented in [10].

\section{Double-sided TFGs with an under-side thin film heater}

The reason of using heated thin film gauges is to heat the gauge substrate in order to increase the temperature range over which the extrapolation to obtain the adiabatic wall temperature and heat transfer coefficient is performed. The first gauge concept presented here is a double-sided thin film gauge with a separate local heating system.

\subsection{Principle of operation}

For an unheated gauge test the initial voltage $V_{\mathrm{i}}$ across each gauge is recorded prior to the run (a few minutes beforehand) to acquire an offset signal at a known component temperature. The initial component wall temperature $T_{\text {wi }}$ is measured at the same time by nearby thermocouples. Given the $\sim 45$ min time passing between tests, isothermal conditions can be assumed. The voltage across a gauge is then recorded during the test time. The change in temperature follows from the change in resistance according to

$$
T-T_{\mathrm{wi}}=\frac{\left(R-R_{\mathrm{i}}\right)\left[1+\alpha\left(T_{\mathrm{wi}}-T_{\mathrm{cal}}\right)\right]}{\alpha R_{\mathrm{i}}}
$$

where $T_{\text {cal }}$ corresponds to the temperature at which the temperature coefficient of resistance $\alpha$ was determined. The gauge

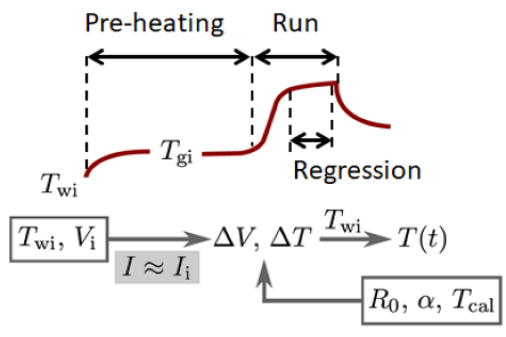

Figure 3. Principle of operation to measure the surface temperature history for a heated thin film gauge arrangement with a separate heating system.

current $I$ stays constant $I \approx I_{\mathrm{i}}$, thus the temperature difference can be expressed as a change in voltage:

$$
T-T_{\mathrm{wi}}=\frac{\left(V-V_{\mathrm{i}}\right)\left[1+\alpha\left(T_{\mathrm{wi}}-T_{\mathrm{cal}}\right)\right]}{\alpha V_{\mathrm{i}}}
$$

and no knowledge of the exact current is required. This calculation is valid for single-sided as well as double-sided thin film gauges.

When taking heat transfer measurements with double-sided gauges with a separate heating source, the temperature of the part is recorded before the heating starts. The heating of the gauge substrate is captured by the increase in voltage from the initial voltage to the voltage recorded just prior to the run. The post-processing is the same as described above. The principle of operation for measuring the surface temperature is illustrated in figure 3.

\subsection{Gauge construction}

The first system investigated to heat the gauge substrate consists of a resistive heater element mounted on a Kapton sheet and placed underneath the thin film gauges on the surface of the part to be measured. The bespoke heater element has the advantages that it provides uniform heating, does not require space in the wall (as a cartridge heater would), and thus does not affect the structural strength of the component, making it a good choice for a heavily cooled vane. However, the disadvantage of this concept is that it requires an additional insulating layer. Figure 4 shows the gauge configuration with an underside thin film heater.

A heater design was developed with a spiral-shaped copper track. The thickness and spacing of the tracks was limited by the accuracy of the etching process to $0.2 \mathrm{~mm}$. An example of a heater design is shown in figure 5 . This heater has a surface area of $525 \mathrm{~mm}^{2}$ and a resistance of $8.1 \Omega$. Depending on the length of the heater tracks and the thickness of the copper, a wide range of resistances can be achieved. The heating power is limited to a temperature range that in practice is observed not to lead to significant changes in the thermal properties of the glue via either outgassing or chemical changes within the glue layer. Limiting the maximum heat flux to $100 \mathrm{~kW} \mathrm{~m}^{-2}$ led to a maximum pre-heating temperature difference of approximately $100 \mathrm{~K}$, or a maximum gauge temperature of approximately $120{ }^{\circ} \mathrm{C}$. At these temperatures the calibration of the 


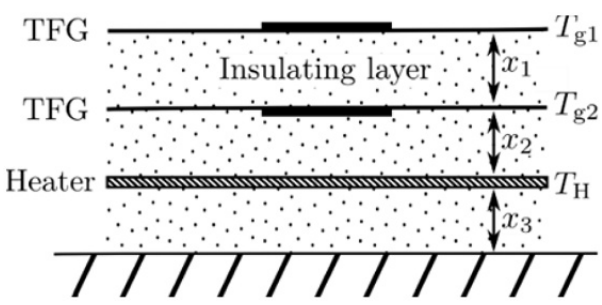

Figure 4. Heated thin film gauge arrangement with an underside thin film heater.

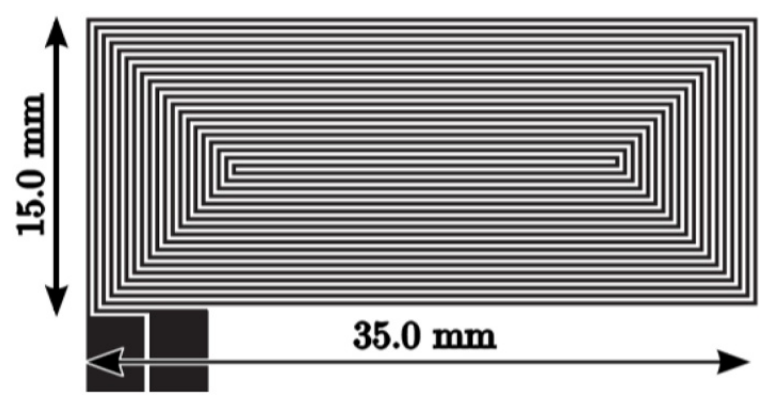

Figure 5. Example of a heater design.

gauge was found to be relatively stable prior to runs in the experimental facility, and essentially unchanged by a number of short duration experiments at elevated temperature. For completeness it is noted that the outgassing process in the glue layer is a complex, highly temperature dependent effect [21], that can be assumed to be ongoing even at low $\left(40{ }^{\circ} \mathrm{C}\right)$ temperatures but at an increasing rate at higher temperatures. In certain applications a further sensitivity study may be deserved, but for our purpose it is sufficient to observe that the calibration is relatively unaffected by this effect in the range of temperatures in which we operated.

\subsection{Modelling and bench test validation}

As the lateral extension of the thin film gauges is small compared to that of the heater element, the heat transfer in the gauge system depicted in figure 4 is considered onedimensional and is described by the following equations:

$$
\begin{gathered}
\dot{q}_{\mathrm{conv}}=h\left(T_{\mathrm{g} 1}-T_{\infty}\right)=k \frac{T_{\mathrm{g} 2}-T_{\mathrm{g} 1}}{x_{1}} \\
k \frac{T_{\mathrm{g} 2}-T_{\mathrm{g} 1}}{x_{1}}=k \frac{T_{\mathrm{H}}-T_{\mathrm{g} 2}}{x_{2}} \\
k \frac{T_{\mathrm{H}}-T_{\mathrm{g} 2}}{x_{2}}=\dot{q}_{\mathrm{H}}-k \frac{T_{\mathrm{H}}-T_{\mathrm{w}}}{x_{3}}
\end{gathered}
$$

where $h$ and $k$ are respectively the surface heat transfer coefficient and the thermal conductivity of the Kapton-glue laminate, and $T_{\infty}$ is the gas temperature, $x_{1}, x_{2}$ and $x_{3}$ the thicknesses of the three Kapton-glue layers, $T_{\mathrm{g} 1}$ and $T_{\mathrm{g} 2}$ the gauge temperatures, and $T_{\mathrm{H}}$ and $T_{\mathrm{w}}$ the heater and wall temperatures respectively.
The heated thin film gauge arrangement with an underside thin film heater element was bench tested over a range of heater current settings. The pre-heating surface temperatures were measured by the double-sided thin film gauges and compared to the modelling results (see figure 6). The current through the heater was kept constant for the duration of each test and increased stepwise from 0 A to $1.6 \mathrm{~A}$. Two consecutive tests were carried out to investigate repeatability. Figure 6 shows the temperature difference relative to the backwall temperature as measured by two thin film gauges. The agreement between two consecutive tests is excellent. The difference between the two thin film gauges is proportional to the total preheating temperature and is at most $2 \mathrm{~K}$. While this temperature difference lies within the expected temperature uncertainty (see section 8) it could also point to slightly uneven heating.

The steady-state model results were obtained for an isothermal top layer, i.e. $T_{\mathrm{g} 1}=T_{\mathrm{g} 2}=T_{\mathrm{H}}$. To account for the heat dissipation into the environment a correlation for the Nusselt number for a surface in natural convection was used. The Nusselt number $\mathrm{Nu}$ can be expressed as a function of Grashof number Gr and Prandtl number Pr [22]:

$$
\mathrm{Nu}=C(\mathrm{GrPr})^{n}
$$

where $C$ and $n$ are two empirical factors, which de-pend on the geometry and the flow conditions. For a heated horizontal plate the Nusselt number can be calculated for a given range of surface temperatures to obtain the convective heat transfer coefficient $h$. It was found that the natural convection, and hence Nusselt number, were very low. Therefore, the gauge substrate is nearly isothermal and the Nusselt number correction is very small. This prediction is in agreement with the observation that the temperatures of the bottom gauges were very similar to those of the top gauges. Overall, the measured temperatures agree well with the model predictions given the experimental uncertainties in backwall temperature and thickness of the insulating layers.

\subsection{Rig testing results}

Transient rig tests were carried out in the Oxford Turbine Research Facility (OTRF). The OTRF is a short duration piston tunnel capable of testing engine-sized high-pressure (HP) turbine stages at engine-representative conditions for aerodynamic and heat transfer measurements. The fundamental operation of this type of facility was first described by Jones et al [23]. The aim of these tests was to demonstrate the accuracy improvement in the regression of heat flux as a function of wall temperature-performed to determine the adiabatic wall temperature and the heat transfer coefficient—obtained by locally heating the substrate.

During this test campaign the OTRF was run without the turbine stage, i.e. with the HP NGV and rotor removed and replaced with a simple annular nozzle configuration. The gauges were placed on the casing endwall, just after what corresponds to the blade over-tip region. These tests were performed under uniform inlet conditions with an inlet total 


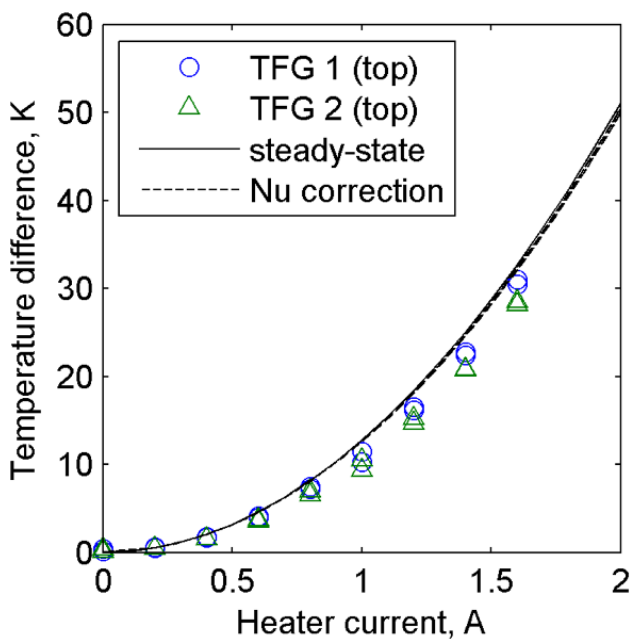

Figure 6. Preheating top gauge temperature differences as a function of heater current for 18 consecutive tests and comparison to analytical results.

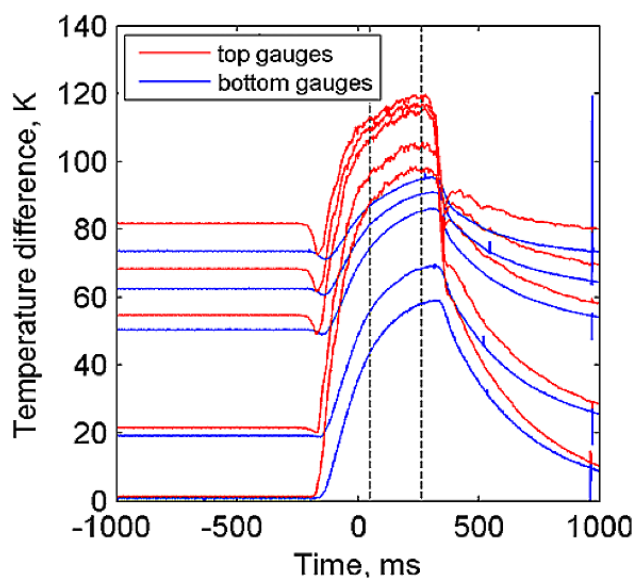

Figure 7. Temperature traces for one double-side gauge with an underside thin film heater (one unheated run and four heated runs).

temperature of $412 \mathrm{~K}$ and Mach number $M=0.9$ at the throat (just upstream of the gauge location).

The temperature traces for one double-sided gauge with an underside thin film heater are shown in figure 7 . The dashed lines indicate the stable run period used for the data regression. The temperature achieved by the gauges before the run depends on the amount of preheating. The heater is switched on a few minutes before the run after taking the measurement of the reference voltage at isothermal conditions. This voltage is used to compute the preheating temperature difference. Note also that the heater was not switched off until the test run was finished. A maximum preheating surface temperature difference of $80 \mathrm{~K}$ was achieved. Although not shown here, at identical heater settings the results were repeatable. The surface temperature drops just before the run when ambient air accumulated in the rig is blown out. The temperatures of top and bottom gauges agree with each other within expected experimental uncertainties and agree well with model predictions.

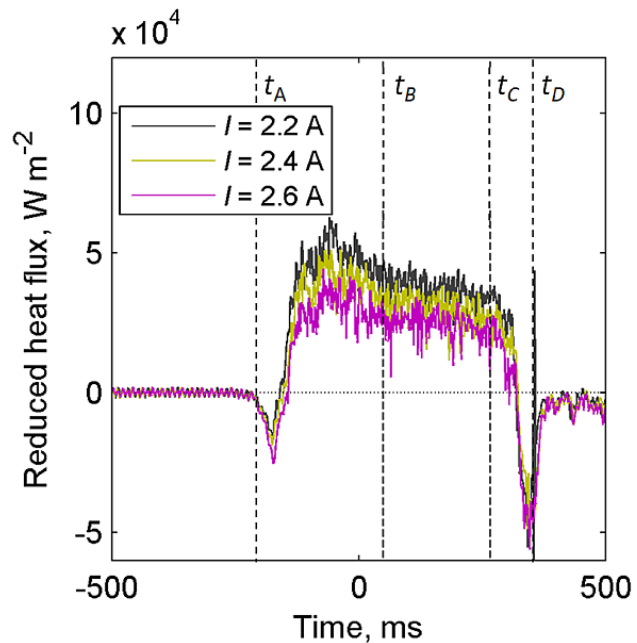

Figure 8. Heat flux traces for one double-sided gauge with an underside heater. Key times as follows: establishment of flow, $t_{A}$; stable period of run used for regression, $t_{B}$ to $t_{C}$; end of run, $t_{D}$.

The convective heat flux is obtained from the temperature history using the impulse response method [19]. The preheating temperature difference is set to zero at the start of the run, because the heat flux resulting from this temperature difference reflects the effect of heating and should therefore be subtracted from all subsequent measurements. This procedure ensures the heat flux is zero at the start of the run. Crucially, uncertainties in the initial temperature difference between top gauge and bottom gauge do not affect the measured convective heat flux.

The reduced heat flux traces for three test runs conducted at different heater current settings are shown in figure 8 . At higher pre-heat temperatures the heat flux during the run decreases and thus the range for the regression is increased in comparison to the unheated double-sided gauges. The regression is performed on data from multiple runs over the stable run period taking into account any changes in the inlet total temperature $T_{01}$ [14]. The regression data for a heated and unheated test case are shown in figure 9. A linear least squares regression method was used (note that Collins et al [14] advocate making the data unbiased in corrected temperature, by resampling the data on a uniform grid of the abscissa). The dashed vertical lines mark the regression range. The adiabatic wall temperature was determined as $425 \mathrm{~K}$ and the heat transfer coefficient as $1397 \mathrm{~W} \mathrm{~m}^{-2} \mathrm{~K}^{-1}$.

The accuracy with which the adiabatic wall temperature can be determined depends on the ratio of the outer wall temperature difference during the stable part of the run $\Delta T_{\mathrm{w}}=T_{\mathrm{w} 2}-T_{\mathrm{w} 1}$ and the difference between the adiabatic wall temperature $T_{\text {aw }}$ and the first (furthest) regression point $T_{\text {w1 }}$. The stability criterion $S$ is introduced, where

$$
S=\frac{\Delta T_{\mathrm{w}}}{T_{\mathrm{aw}}-T_{\mathrm{w} 1}}
$$

For the unheated case $S$ is as low as 0.36 . For the heated case the value for the stability criterion rises to 0.79 . The best 


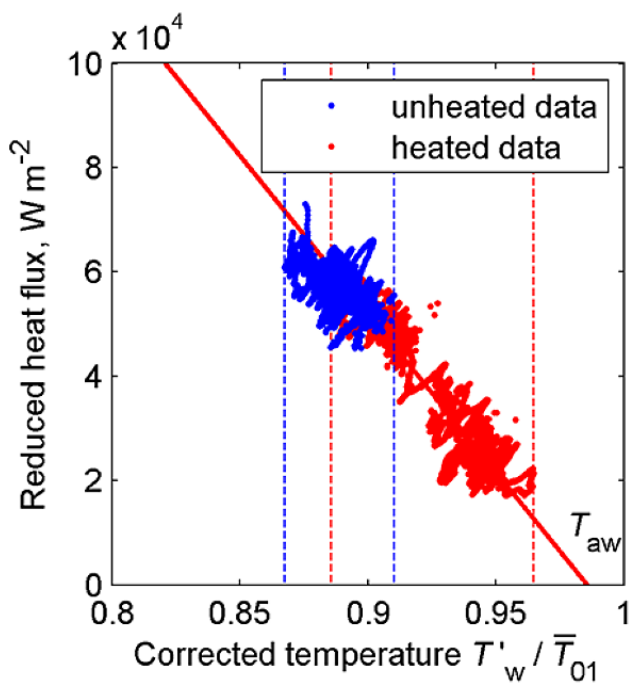

Figure 9. Regression data for one double-sided gauge with an underside heater compared to unheated results.

results are obtained with a combination of unheated and heated data $(S=0.82)$.

\section{Self-heating double-sided thin film gauges}

The concept for self-heating gauges is to use heat transfer gauges in a self-heating mode to change the surface temperature-between a number of tests-prior to a run. To exploit the ohmic heating of the gauges, they are supplied with a higher current than usually used for the heat transfer measurements. In such a set-up the surface temperature will be higher at the position of the gauges, leading to non-uniform surface temperature prior to a run. Provided this difference is small in comparison to the difference of temperature between gas and surface, the correction for the so-called heat-island effect [24] can be assumed to be modest. We discuss this in more detail when considering the uncertainty analysis associated with the gauges.

At the beginning of the test the surface is in thermal equilibrium, i.e. the electrical power converted into heat by the thin film gauges equals the heat losses via conduction and convection. Since the conduction field after the beginning of the run is expected to remain nearly constant, it is possible to infer the change in convective surface heat flux during the run by measuring the surface temperature with the thin film gauges. The local heat flux into the surface can be calculated using a conventional 1D conduction analysis, provided the convective heat flux does not vary significantly between different points on the preheated surface. The measured heat transfer rate is equal to the surface heat flux minus the known pre-run heat flux.

\subsection{Principle of operation}

For thin film gauges with variable heating current through the gauge, the equation for heated gauges with an underside thin film heater cannot be used because both the current and

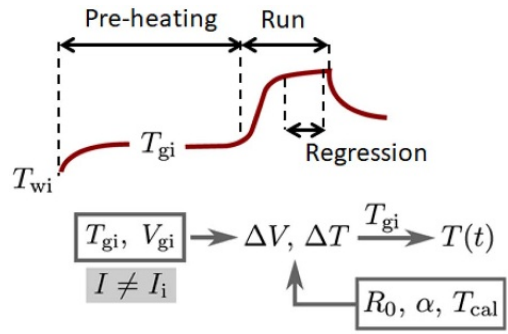

Figure 10. Principle of operation to measure the surface temperature history for self-heating thin film gauges.

the gauge temperature change during preheating. Therefore, heat transfer measurements require additional information on the initial gauge temperature $T_{\text {gi }}$. This temperature cannot be measured directly in the rig due to access limitations, but it can be infer-red from external information such as bench test data or thermal models. Once $T_{\text {gi }}$ is known and the corresponding voltage $V_{\text {gi }}$ has been measured, any subsequent change in temperature can be calculated:

$$
\Delta T=T-T_{\mathrm{gi}}=\frac{\left(V-V_{\mathrm{gi}}\right)\left[1+\alpha\left(T_{\mathrm{gi}}-T_{\mathrm{cal}}\right)\right]}{\alpha V_{\mathrm{gi}}}
$$

In this study, the current through the bottom gauge was kept constant and at a level that did not cause any ohmic heating. The temperature increase of the bottom gauges can therefore be calculated as for the heated gauges with an underside heater, where the initial temperature of the part is recorded under isothermal conditions before the heating starts. Using an accurate model of the heat conduction between the two gauges, it is then possible to derive the temperature of the top gauge from the bottom gauge temperature, provided the two gauges are very well aligned.

In summary, for the self-heating gauges the top and bottom temperatures are calculated in two different ways. The bottom gauge temperature trace follows from the initial temperature and the corresponding change in voltage, as for a gauge with an underside heater. However, the current through the top gauge is increased after the reference measurement is taken and knowledge of the initial gauge temperature is required to obtain the temperature trace (see figure 10). The problem of determining the initial gauge temperature will be further discussed below.

\subsection{Gauge construction}

In this case the gauges self-heat by ohmic heating, hence the gauge configuration is identical to that of a conventional double-sided thin film gauge (see figure 11), with the gauges position one above the other. In comparison to the doublesided thin film gauges with an underside thin film heater, the total thickness of the package is reduced by the thickness of one additional insulating layer, which constitutes about one third of the overall assembly.

The heating of the gauges depends on both the current through the gauge and the resistance and area of the gauge. The change in gauge resistance with temperature can be calculated 


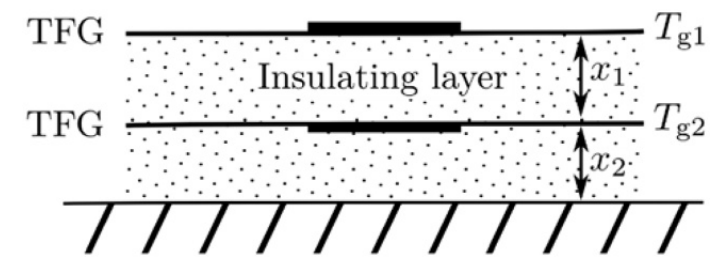

Figure 11. Self-heating gauges.

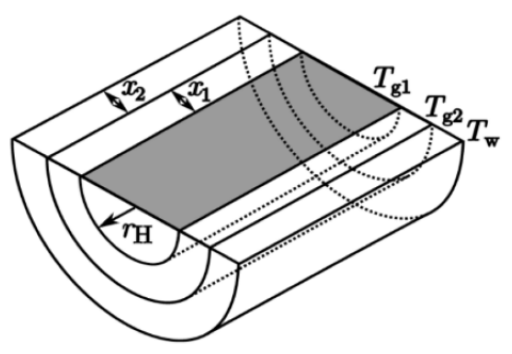

Figure 12. $2 \mathrm{D}$ analogue of the gauge heat transfer system.

from the temperature coefficient of resistance. As with the heater element, the heating power was limited to $100 \mathrm{~kW} \mathrm{~m}^{-2}$ to avoid material property changes associated with outgassing. The variable current supply for the heated gauges is described in the appendix B.

\subsection{Modelling and validation}

For the self-heating gauges it is necessary to determine the initial top gauge temperature, which is difficult to measure directly. If this temperature is to be inferred from another measured temperature such as the bottom gauge temperature or the wall temperature, it is necessary to describe the conduction in the gauge substrate. The heating heat flux can be calculated from the known voltage and resistance of the top gauge $\dot{q}_{\mathrm{H}}=V^{2} /\left(R A_{\mathrm{g}}\right)$, where $A_{\mathrm{g}}$ is the platinum surface area. If the preheating has reached steady-state before the run, the temperature of the top gauge then follows from thermal conduction.

For a gauge track width of $0.3 \mathrm{~mm}$, and an insulating layer thickness of $100 \mu \mathrm{m}$, an analogue in cylindrical co-ordinates is possible that is a relatively close approximation of the physics of the system. This is represented in figure 12. In this system analogue, the radius of the heating source $r_{H}$ is set so that the surface area per unit length of the inner cylinder is equal to the surface area per unit length of the gauge track. The heating power per unit length is set to that which would be developed by the gauge. The thermal mass is set to be zero and the thermal conductivity to be infinite. This imposes something close to the correct physical boundary condition at the interface between the inner cylinder and the first shell, the platinum having a thickness of less than $1 \mu \mathrm{m}$. The first and second shells are set to have the same thermal properties as the two Kapton-glue layers (physical thicknesses $x_{1}$ and $x_{2}$ respectively) and for a radius $r>x_{1}+x_{2}+r_{H}$ the thermal properties of the lower substrate are used. Despite being a relatively crude model, the predictions are in reasonable agreement with the experimental data.

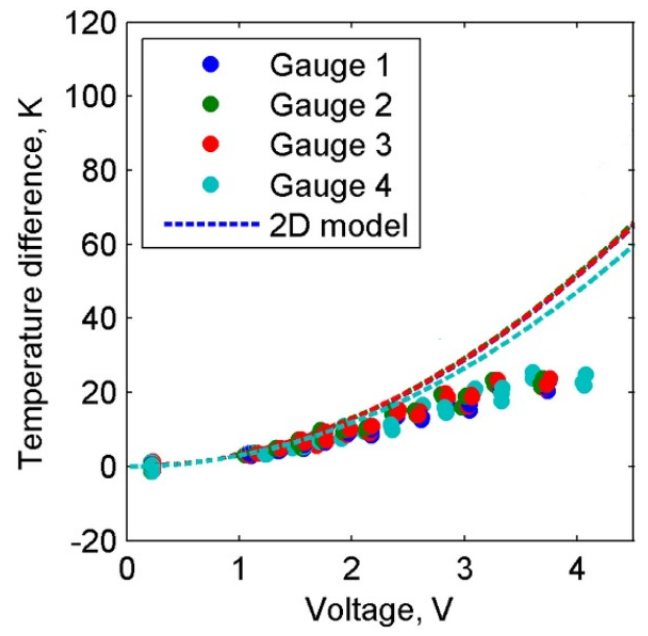

Figure 13. Pre-heating temperature difference between the bottom gauge and the wall for a range of top gauge voltage settings. Results from a $2 \mathrm{D}$ analogue model are also given.

Figure 13 shows the preheating temperature difference between the bottom gauge and wall as measured in the OTRF for a range of top gauge voltages, compared to the 2D analogue. This data serves to validate the thermal model that is used to calculate the initial top gauge temperature for a known heating heat flux (the upper surfaces losses into the rig at vacuum prior to the run are limited to a small radiative load).

The agreement between the four self-heating gauges is good. Up to $2.5 \mathrm{~V}$ the agreement between the $2 \mathrm{D}$ model prediction and the experimental bottom gauge temperature differences is good (within experimental uncertainties). At higher voltages the bottom gauge temperature deviates from the prediction, with a flattening of the temperature curve. This effect can be partially explained by the increase in gauge resistance with increasing gauge temperature, which leads to a decrease in the heating heat flux. This effect was taken into account in post-processing. In a later section we perform an uncertainty analysis, in which we account for the error in temperature difference arising from the need to model the pre-run temperature of the top gauge.

For completeness we note that it would be desirable to further reduced the error associated with this modelling process. It is recommended that in future work investigators use a full 3D model of the gauge system to improve modelling accuracy, and explore the possibility of improved electronics to allow direct measurement of gauge temperature. For our purpose we are concerned with proof-of-concept.

\subsection{Rig testing results}

Identical transient rig tests were carried out in the OTRF with self-heating double-sided thin film gauges as described previously for the double-sided gauges with an underside heater. Both types of gauges were mounted at the same axial location in the annular nozzle, and as no circumferential variation in the flow was expected, this allowed a direct comparison between the two configurations. 


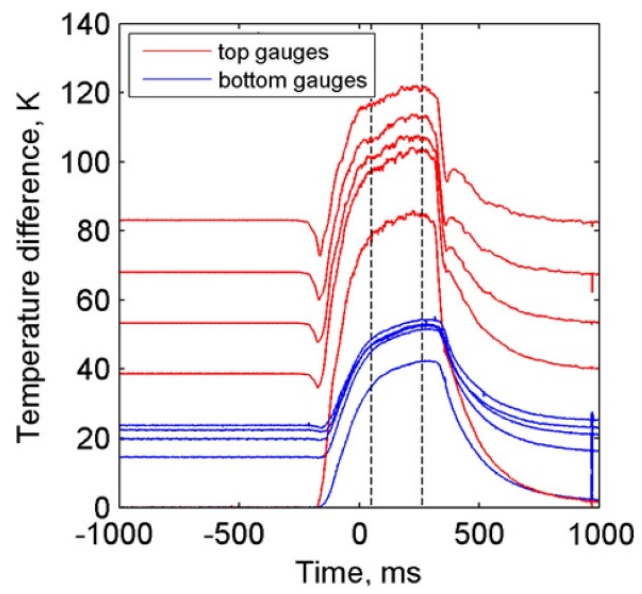

Figure 14. Temperature traces for one self-heating gauge (one unheated run and four heated runs).

The temperature traces for the same double-sided gauge with a self-heated top gauge tested over a range of heating power settings are shown in figure 14. Preheating surface temperature differences of up to $80 \mathrm{~K}$ were achieved. As for the double-sided thin film gauges with an underside heater, the heat flux is calculated using the impulse response method with the temperature difference set to zero at the start of the run. The resulting reduced heat flux is equal to the convective heat flux alone, i.e. the surface heat flux minus the pre-run heat flux. With increasing gauge current, the surface temperature increases and the heat flux decreases.

The regression data for a heated and unheated test case from one self-heating double-sided thin film gauge are presented in figure 15. The heated results agree well with those of the unheated double-sided thin film gauge. A wide temperature range is covered with the heated runs and a very good regression is achieved, which enables the adiabatic wall temperature and the heat transfer coefficient to be determined separately.

The heat transfer results between the two heated double-sided gauge configurations were very similar. The adiabatic wall temperature and heat transfer coefficient measured with the self-heating double-sided gauges were $417 \mathrm{~K}$ and $1333 \mathrm{~W} \mathrm{~m}^{-2} \mathrm{~K}^{-1}$ respectively. These compared well to the values of $425 \mathrm{~K}^{-1}$ and $1397 \mathrm{~W} \mathrm{~m}^{-2} \mathrm{~K}^{-1}$ measured using the gauges with an underside heater.

The improvement in the stability of the regression can again be quantified with the stability criterion introduced above for the double-sided thin film gauges with an underside heater. For the unheated results the stability criterion $S$ is 0.31 , whereas for the heated results it is 0.96 . A combination of unheated and heated runs yields the highest value $(S=0.97)$.

\section{Uncertainty analysis}

To estimate the uncertainty of the heat transfer coefficient, it is instructive to consider two discrete measurements of the wall temperature, $T_{1}$ and $T_{2}$, and the corresponding heat fluxes $\dot{q}_{1}$

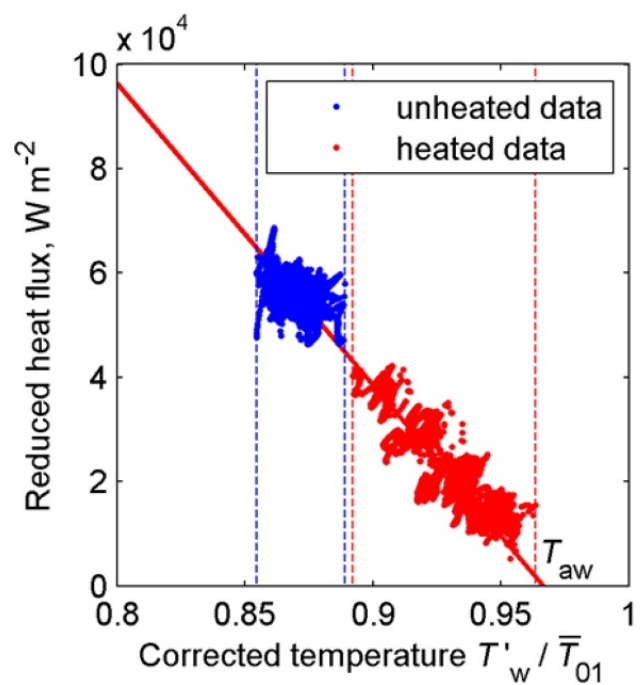

Figure 15. Regression data for one self-heating double-sided gauge compared to results from unheated double-sided thin film gauges.

Table 1. Summary of uncertainties for three techniques.

\begin{tabular}{lccc}
\hline Variable & Unheated & Underside heater & Self-heating \\
\hline$T(\mathrm{~K})$ & $360 \pm 1.8$ & $420 \pm 4.5$ & $420 \pm 15$ \\
$T_{\mathrm{i}}(\mathrm{K})$ & $290 \pm 1.0$ & $370 \pm 3.7$ & $370 \pm 15$ \\
$\Delta T(\mathrm{~K})$ & $70 \pm 1.5$ & $50 \pm 2.6$ & $50 \pm 2.6$ \\
$\dot{q} \times 10^{4}\left(\mathrm{~W} \mathrm{~m}^{-2}\right)$ & $6.0 \pm 0.3$ & $1.5 \pm 0.1$ & $1.5 \pm 0.1$ \\
$h\left(\mathrm{~W} \mathrm{~m}^{-2} \mathrm{~K}^{-1}\right)$ & - & $750 \pm 80$ & $750 \pm 196$ \\
$T_{\text {aw }}(\mathrm{K})$ & - & $440 \pm 7.0$ & $440 \pm 20$ \\
\hline
\end{tabular}

and $\dot{q}_{2}$. The heat transfer coefficient is then given by

$$
h=\frac{\dot{q}_{2}-\dot{q}_{1}}{T_{2}-T_{1}}
$$

The achievable temperature differences during a single run are rather small and statistical uncertainties are typically very large. It is therefore desirable to combine heated and unheated runs. While this approach leads to larger systematic uncertainties due to the need to compare runs with different initial wall temperatures, it significantly increases the range of $T_{\mathrm{w}}$ and hence improves the reliability of the linear regression. The resulting uncertainties in $h$ can be calculated for a specific example, which is presented in table 1 . For this example he measurements of $T_{1}$ and $\dot{q}_{1}$ correspond to the unheated case, whereas $T_{2}$ and $\dot{q}_{2}$ result from one of the heated cases. The adiabatic wall temperature is determined from a linear extrapolation of the heat flux results for a set of different values of $T_{\mathrm{w}}$. The given temperatures and heat fluxes are representative for the experimental data. With the current thermal models for the self-heating gauges, the uncertainties affecting the determination of the initial gauge temperature are high, and the gauges with an underside heater achieve more accurate results.

For completeness, we should note that the measured heat transfer coefficient, though to first order independent of the local gas-to-wall temperature ratio, does depend on the temperature distribution (in space) of the wall upstream of the point of interest. An isothermal wall is only realised in certain 
well-controlled experiments, and-perhaps—approximately realised for particular surfaces in turbomachinery (result of particular optimisation). Thus, for many real engine components, and in many real experiments, there are particular wall temperature distributions. These may not be matched to each other, leading to dissimilarity in the temperature distribution within the thermal boundary layers in the two environments. For quantitative comparisons between test facility and engine, scaling of results for the so-called heat island effect is required. A practical method for performing this correction in given in [24]. For the first technique described in this paper, the effect can largely be mitigated by ensuring the heater extends to the leading edge of the part. For the second technique the effect is more difficult to mitigate and corrections may need to be employed.

\section{Conclusions}

Two methods have been presented to reduce uncertainty in the transient heat transfer technique using thin film gauges. Both methods make use of double-sided thin film gauges to measure the transient heat flux into a surface by means of the unsteady temperature rise that occurs in short-duration testing. Uncertainty is reduced over conventional techniques by extending the range of temperature over which the regression (of heat flux as a function of wall temperature) is performed. This is achieved by pre-heating the surface. The first technique uses a thin film heater as an additional layer-under the double layer gauge-within the substrate. The second technique uses the gauge as a self-heater. We show both methods improve the uncertainty in the experimentally determined adiabatic wall temperature and the corresponding heat transfer coefficient. Both techniques have the advantage that they can be installed locally, removing the need for complex pre-heating systems for entire components. In certain applications the proposed techniques should represent an improvement over prior methods.

\section{Acknowledgments}

The authors would like to thank Martin Oldfield for the help in modifying the heat transfer amplifier for the self-heating gauges. The thin film gauges were designed with the input from Matthew Collins. Many thanks to Trevor Godfrey for manufacturing the gauges and to David Cardwell and Sunny Chana for conducting the experiments in the OTRF.

\section{Appendix A: Impulse response processing of transient heat transfer signals}

Historically, the experimental heat transfer rate was obtained from the surface temperature signal using numerical approximations to the solutions of the linear differential equations relating the two. The impulse response method, in contrast, only uses known pairs of exact solutions, such as the temperature response to a step in heat flux, to derive a sampled approximation of the impulse response of the gauge system [19]. This impulse response is then used as a finite impulse response digital filter to convert the surface temperature signal into a heat transfer rate. It is enough to calculate the impulse response once for each set of gauge parameters, making this processing of surface temperature signals computationally very efficient. This method and the corresponding filter routines have been extended to two-layer substrate gauges and doublesided gauges [19].

\section{Appendix B: Use of HTA3 amplifier and modification for higher currents}

The HTA3 thin film signal conditioning amplifier is used as a current source for the thin film gauges and to record the heat transfer signal. In order to achieve the higher currents necessary for using the HTA3 amplifier with self-heating gauges, the original maximum current of $20 \mathrm{~mA}$ had to be increased. This change required a modification of the high constant current supply to allow for thin film gauge currents up to $150 \mathrm{~mA}$ [25].

\section{ORCID iD}

Thomas Povey (D) https://orcid.org/0000-0001-8977-9644

\section{References}

[1] Childs P R N, Greenwood J R and Long C A 1999 Heat flux measurement techniques Proc. Inst. Mech. Eng. C 213 655-77

[2] Schultz D L and Jones T V 1973 Heat Transfer Measurements in Short Duration Hypersonic Facilities (Springfield, Va: NATO Advanced Group for Aerospace Research and Development) (AG-165)

[3] Epstein A H, Guenette G R, Norton R J G and Yuzhang C 1986 High-frequency response heat-flux gauge Rev. Sci. Instrum. 57 639-49

[4] Guo S M, Spencer M C, Lock G D, Jones T V and Harvey N W 1995 The application of thin film gauges on flexible plastic substrates to the gas turbine situation Proc. ASME 1995 Int. Gas Turbine and Aeroengine Congress and Exposition. Volume 4: Heat Transfer; Electric Power; Industrial and Cogeneration (Houston, Texas, USA, 5-8 June) V004T09A049 (ASME) (https://doi.org/10.1115/ 95-GT-357)

[5] Ainsworth R W, Allen J L, Davies M R D, Doorly J E, Forth C J P,, Hilditch M A, Oldfield M L G and Sheard A G 1989 Developments in instrumentation and processing for transient heat transfer measurement in a full-stage model turbine ASME J. Turbomach. 111 20-27

[6] Jones T V 1995 The thin film heat transfer gauges - a history and new developments 4th National UK Heat Transfer Conf., IMechE Conf. Trans. (Manchester, UK, 26-27 September)

[7] Piccini E, Guo S M and Jones T V 2000 The development of a new direct-heat-flux gauge for heat transfer facilities Meas. Sci. Technol. 11 342-9

[8] Anthony R J, Oldfield M L G, Jones T V and LaGraff J E 1999 Development of high-density arrays of thin film heat 
transfer gauges 5th ASME/JSME Thermal Engineering Joint Conf. (San Diego, CA) (AJTE 99-6159)

[9] Thorpe S J, Yoshino S, Ainsworth R W and Harvey N W 2004 Improved fast response heat transfer instrumentation for short-duration wind tunnels Meas. Sci. Technol. 15 1897-909

[10] Collins M, Chana K S and Povey T 2015 New technique for the fabrication of miniature thin film heat flux gauges $J$. Meas. Sci. Technol. 26025303

[11] Lyons P R A and Gai S L 1988 A method for the accurate determination of the thermal product $(\rho \mathrm{ck})^{1 / 2}$ for thin film heat transfer or surface thermocouple gauges $J$. Phys. E 21445

[12] Doorly J E 1985 The development of a heat transfer measurement technique for application to rotating turbine blades DPhil Thesis University of Oxford

[13] Xue S, Roy A, Ng W F and Ekkad S V 2015 A novel transient technique to determine recovery temperature, heat transfer coefficient, and film cooling effectiveness simultaneously in a transonic turbine cascade ASME J. Therm. Sci. Eng. Appl. 7011016

[14] Collins M, Chana K S and Povey T 2016 Improved methodologies for time-resolved heat transfer measurements, demonstrated on an unshrouded transonic turbine casing ASME J. Turbomach. 138111007

[15] Thorpe S J, Yoshino S, Ainsworth R W and Harvey N W 2004 An investigation of the heat transfer and static pressure on the over-tip casing wall of an axial turbine operating at engine representative flow conditions. (I). Time-mean results Int. J. Heat Fluid Flow 25 933-44

[16] Thorpe S J, Yoshino S, Ainsworth R W and Harvey N W 2004 An investigation of the heat transfer and static pressure on the over-tip casing wall of an axial turbine operating at engine representative flow conditions. (II). Time-resolved results Int. J. Heat Fluid Flow 25 945-60

[17] Doorly J E and Oldfield M L G 1987 The theory of advanced multi-layer thin film heat transfer gauges Int. J. Heat Mass Transfer 30 1159-68

[18] Oldfield M L G, Burd H J and Doe N G 1984 Design of wide-bandwidth analogue circuits for heat transfer instrumentation in transient tunnels Heat and Mass Transfer in Rotating Machinery (Washington, DC: Hemisphere Publishing Corp.) pp 233-258

[19] Oldfield M L G 2008 Impulse response processing of transient heat transfer gauge signals $J$. Turbomach. 130021023

[20] Usandizaga I 2016 Investigations of improved heat transfer instrumentation for cooled turbine stages $P h D$ Thesis University of Oxford

[21] Laikhtman A, Verker R, Gouzman I, Noter Y and Grossman E 2006 Outgassing effects of kapton acrylic adhesive tape European Space Agency Special Publication ESA SP Issue $6166 \mathrm{p}$

[22] Howatson A M, Lund P G and Todd J D 2009 Engineering Tables and Data (University of Oxford) (internal publication)

[23] Jones T V, Schultz D L and Hendley A D 1973 On the flow in an isentropic light piston tunnel Reports and Memoranda Aeronautical Research Council, Great Britain

[24] Moffat R J, Eaton J K and Mukerji D 2000 A general method for calculating the heat island correction and uncertainties for button gauges J. Meas. Sci. Technol. 11 920-32

[25] Oldfield M L G and Beard P F 2014 HTA3 Heat Transfer Amplifier 3 Version 1.5 (University of Oxford) (internal report) 